TTR

Traduction, terminologie, re?daction

\title{
Le don et l'abandon : la trace de la traduction dans la blanche écriture du concept
}

\section{Laurent Lamy}

Volume 12, numéro 2, 2e semestre 1999

Poésie, cognition, traduction II - Autour d'un poème de W. H. Auden Poetry, Cognition, Translation II — On a Poem by W. H. Auden

URI : https://id.erudit.org/iderudit/037376ar

DOI : https://doi.org/10.7202/037376ar

Aller au sommaire du numéro

\section{Éditeur(s)}

Association canadienne de traductologie

ISSN

0835-8443 (imprimé)

1708-2188 (numérique)

Découvrir la revue

Citer cet article

Lamy, L. (1999). Le don et l'abandon : la trace de la traduction dans la blanche écriture du concept. TTR, 12(2), 131-158. https://doi.org/10.7202/037376ar
Résumé de l'article

Le don et l'abandon : la trace de la traduction dans la blanche écriture du concept - Cette étude met en question l'ascendant du concept dans le domaine de l'expression ou, plus généralement, celui des « arts du sens ». L'impossibilité qui frappe la nécessité même de la traduction comme vecteur de la signification, loin de l'aliéner, l'instruit plutôt comme agent créateur d'une dimension de sens dont l'ouverture, virtuellement infinie, est intimement liée à la finitude qui s'attache au partage de la condition humaine. C'est là la limite du concept et le plan de démarcation de toute poétique en regard de la normativité inhérente à l'exercice du langage. C'est aussi la frontière qu'explore la traduction, qui ne saurait alors être reléguée à une fonction ancillaire en vertu de laquelle il lui incomberait simplement de préserver l'univocité du signifié (concept) commandant la translation idyllique entre une langue-source et une langue-cible. La traduction est par essence "performative ", c'est-à-dire qu'elle produit du sens. Ainsi fait-elle brèche vers une dimension d'universalité qui n'est pas d'abord subordonnée à l'unité du concept, mais se ressource et trouve son épanouissement dans l'unicité de la forme, celle d'une langue dont l'incomplétude est non seulement avérée, mais sollicitée dans son rapport à l'étranger. Il ne lui appartient pas de communiquer quelque chose, mais de révéler l'essence même de la communicabilité.

Tous droits réservés @ TTR: traduction, terminologie, rédaction — Les auteurs, Ce document est protégé par la loi sur le droit d’auteur. L’utilisation des 1999

services d'Érudit (y compris la reproduction) est assujettie à sa politique d'utilisation que vous pouvez consulter en ligne.

https://apropos.erudit.org/fr/usagers/politique-dutilisation/ 


\title{
Le don et l'abandon : la trace de la traduction dans la blanche écriture du concept*
}

\section{Laurent Lamy}

\author{
WEGGEBEIZT vom \\ Strahlenwind deiner Sprache \\ das bunte Gerede des An- \\ erlebten - das hundert- \\ züngige Mein- \\ gedicht, das Genicht. \\ RONGÉ par \\ le souffle irradié de ta parole \\ le babil criard du sans- \\ péril - aux cents- \\ langues mon parjuré de \\ poème, le moins que rien.
}

Paul Celan

\footnotetext{
-Version intégrale d'une communication prononcée à l'Université McGill, le 17 mai 1996, dans le cadre du colloque "La traduction dans le champ des sciences humaines ", à l'invitation des professeurs Annick Chapdelaine, du Département de langue et littérature françaises de l'Université McGill, et Alexis Nouss, du Département de linguistique et de traduction de l'Université de Montréal.
} 
Vous entendez maintenant des chants

parfaits.

\section{Giraldo di Borneill (poète provençal du XIII ${ }^{\text {ième }}$ siècle)}

Une langue passe-t-elle dans l'autre, va-t-elle vers l'autre, en appelle-telle à cette autre qui n'est pas autre qu'elle, mais autre sans plus? Une langue n'est-elle pas destinée à passer, à migrer vers une frontière qu'elle ne saurait définir de l'intérieur, car ce qui lui est propre non moins que ce qui lui est étranger échappe à la maîtrise de son exercice, à ce qui constituerait sa juridiction ou son aire de rayonnement? N'estelle pas déjà orpheline de toute origine à laquelle l'archéologie des signes saurait la reconduire?

Marquée par un pareil abandon, qui menace aussi de la frapper de déshérence quant à son futur, une langue n'est-elle pas déjà, in nuce, en souffrance de sa traduction, comme la pensée qui s'y ressource, une pensée qui vient au jour dans cette langue qui n'est pas, qui n'existe pas en dehors de son usage par un mortel, jamais ne pourra fonder sa propre existence, mais seulement fouir, s'enfoncer toujours davantage au ccur de l'élément où elle naît pour en oblitérer la trace, occulter les douleurs de son propre enfantement? Cet élément est la langue, dans sa livraison plurale, que le mythe associe à la diaspora post-babélienne. Nous y sommes livrés, depuis toujours. Mais cela ne se conçoit pas, ne « tombe » pas sous le concept.

On n'a guère de mérite à constater que l'on ne peut s'expliquer le langage, sur lui ou avec lui, que dans la langue, non pas à travers elle mais en elle - l'une des multiples langues dont nous ne pouvons pas même dire qu'elles sont à notre disposition, puisque nous n'avons jamais évolué en dehors de l'une d'entre elles.

(Cette dernière remarque est aussi triviale qu'insondable, triviale parce qu'insondable : on ne saurait réduire notre rapport à la langue ou, si l'on prefere, notre inscription en elle, à une simple fonction instrumentale. Nous ne faisons qu'un avec la langue: il n'est de moment où nous soyons hors d'elle pour ensuite nous en prévaloir comme moyen à l'aide duquel acheminer le contenu de l'acte où nous désirons signifier quelque chose. Le cri du nourrisson dirigé vers le sein 
de la mère ou de la nourrice n'est pas instrumental : le nourrisson est tout entier ce cri. Le langage n'est pas un moyen mais un milieu, une forme de vie.)

Le vaste spectre des ressources expressives s'attachant à l'ingénierie des influx sensoriels, à la domestication du champ visuel, à la gestuelle ou à la symbolique corporelle ainsi qu'à la complexité des formes générées par les diverses grammaires chorégraphiques, ne diminue en rien notre singulière et pourtant si coutumière appartenance au règne de la langue.

Le partage du règne de la langue est aussi celui de la mort qui, d'un point de vue génétique et évolutionnaire, est congénital à la sélection de la reproduction sexuée. Le don de la langue est inexorablement lié à l'abandon de l'individualité, au sacrifice de l'individu au profit de l'espèce et, par conséquent, à la rançon de finitude, qui fait que le sujet parlant est toujours le mortel.

C'est ce que Rilke a saisi en toutes lettres, ce qu'il a bien en vue dans la strophe finale de sa huitième Élégie de Duino, lorsqu'il demande :

Wer hat uns also umgedreht, dass wir, was wir auch tun, in jener Haltung sind von einem, welcher fortgeht?

Qui donc nous a inversés de la sorte que, quoi que nous fassions, nous ayons la contenance de quelqu'un qui s'éloigne?

À jamais inquisiteurs du « quoi », à jamais orphelins du « qui », nous avons trouvé refuge dans la parole. Nous avons déserté l'Ouvert qui désormais nous inquiète, nous déserte : où donc s'abriter de la mort qui nous habite? "Ainsi vivons-nous, écrit Rilke, sans fin prenant congé - so leben wir und nehmen immer Abschied" (trad. Roger Munier, pp. 60-61).

Nous avons perdu ne serait-ce que la trace de l'immensité qui jadis abritait l'élan de la créature dans la magnitude incirconscrite de

" Rainer Maria Rilke, "Huitième Élégie de Duino ", trad. par Roger Munier, dans Le Nouveau Commerce, $\mathrm{N}^{\text {os }}$ 21-22, printemps 1972, pp. 58-61. 
l'Ouvert - das Offene. La seule magnitude qui nous échoit est celle qui répond à la métrique dés-astrée qui arpente l'encre de la nuit criblée de lueurs d'astres fossiles, à l'œil cyclopéen qui épie leurs sémaphores comme des runes laissées à l'abandon dans l'antichambre du néant - piégés que nous sommes par la lumière que nous avons tenté de piéger. Le libre animal, das freie Tier, libre de la mort, l'élan premier de la créature s'est involué en nous, s'est arrêté à la figure du monde où règne la mort. N'est-il pas aussitôt arraché à la mamelle de sa nourrice, que déjà nous plions le jeune enfant, le petit d'homme, le brisons, le soustrayons à la ferveur prodigue de ses gymnopédies, à l'inconstance souveraine de son regard plongé dans l'Ouvert, fasciné par l'aura de la forme qui obéit encore à la réserve secrète de l'inconnu qui règne dans l'atelier des songes :

[...] denn schon das frühe Kind

wenden wir um und zwingens, dass es rückwärts

Gestaltung sehe, nicht das Offne, das

im Tiergesicht so tief ist. Frei von Tod.

[...] car le jeune enfant déjà

nous le ployons et le contraignons pour qu'il voie en deçà

ce qui a figure et non l'Ouvert,

si profond dans la vue animale. Libre de la mort.

(trad. Roger Munier, pp. 54-55)

Nous qui savons, n'avons plus accès à cela qui déjà, depuis toujours, nous abritait et qui désormais ne laisse de nous déserter parce que nous l'avons déserté :

Wir haben nie, nicht einen einzigen Tag, den reinen Raum vor uns, in den die Blumen unendlich aufgehn. Immer ist es Welt und niemals Nirgends ohne Nicht : das Reine Unüberwachte, das man atmet und unendlich weiss und nicht begehrt. Als Kind verliert sich eins im Stilln an dies und wird gerüttelt. Oder jener stirbt und ists. Denn nah am Tod sieht man den Tod nicht mehr und starrt hinaus, vielleicht mit grossem Tierblick. 
Nous, jamais nous n'avons, pas même un seul jour, le pur espace devant nous, en quoi les fleurs sans fin éclosent. C'est toujours le monde et jamais Nulle part sans Rien : le Pur, l'Inviolé, que l'on respire et que sans fin l'on sait, de lui n'ayant nul désir. Enfant, quelqu'un s'y perd dans le silence et en est ébranlé. Un autre meurt et il est cela.

Car proche de la mort on ne voit plus la mort et la vue se fixe au-delà, peut-être en un grand regard

[ animal.

(trad. Roger Munier, pp. 54-57)

La perte à jamais consommée, irréversible, de ce regard sans frontières, originellement affranchi de la syncope, de la césure, de la limite qui nous retient captifs, secrètement sertie dans le halètement du souffle où se nouent le deuil interminable des rites de l'enfance et l'apprentissage d'une agonie qui ne laisse de venir, qui déjà nous serre au plus près, c'est là, nous dit Rilke, " ce qu'on appelle destin : être en face/et cela seul et toujours être en face - Dieses heisst Schicksal: gegenüber sein/und nichts als das und immer gegenüber " (trad. Roger Munier, pp. 56-57).

Transfuge de l'Ouvert, la créature absorbée par la figure du monde, par le chiffre insatiable de ses tribulations, n'a pour seul refuge dans son exil aux confins de sa nuit, que la félicité intangible, furtive, de la parole qui porte en elle le sort de l'équivoque : parole de vie, parole de mort.

Or la vie m'a été donnée par autrui, et de la mort jamais je ne m'approcherai que dans la relation d'inconnu qui se dessine à l'approche de l'autre homme, dans la nudité de son visage, cette abstraction inouie pétrie de chair et de souffle qui déjà s'absente hors des horizons de ce monde. La parole trahit toujours l'approche d'un seuil, la mesure de l'abandon qui m'est signifié dans le visage de l'autre homme. Avoir la contenance de quelqu'un qui s'éloigne, sans fin prenant congé, est le fait de la créature qui habite la parole, qui ne peut déserter le lieu de son assignation, se dérober à la rigueur du commandement qui déjà l'inscrit dans l'espace ouvert par l'appel de l'autre, dans la lointaine proximité du visage où " passe » la trace de 
l'Infini, celle d'un "passé qui ne fut jamais présent ", selon le mot d'Emmanuel Lévinas.

Le visage qui déchire l'horizon à hauteur d'homme, lui ou elle qui m'interpelle, le premier venu, vient de la même région que la mort et m'enjoint comme tel, m'oblige déjà, moi et moi seul, malgré moi.

Mais seul le silence peut traduire cela.

Cela ne peut qu'être tu, et on y trouve, dans l'exclusive de toute possibilité d'énonciation, le germe de l'éthique. Toute parole proférée, celle-là même qui " guérit ", porte en elle la semence de la mort.

(C'est pourquoi, comme Walter Benjamin l'a fort bien intuitionné, le passage à la parole articulée, à la division entre sens et référence, bref à I'horizontalité de la phrase par contraste avec la verticalité intensive du vocable, marque l'avènement du droit, du règne de la loi, la sanction du jugement - ce que d'aucuns parmi les Anciens ont désigné comme la " chute originelle ».)

La langue, qui permet de concevoir quelque chose, ne se conçoit pas. La langue, pour tout ce qu'elle permet de communiquer, ne se communique pas elle-même, à l'instar du sens, qui n'est pas, qui ne correspond à rien qui soit, mais se produit, advient comme l'événement du communicable, la communicabilité même, l'épreuve de sa limite, la splendeur de sa mendicité. C'est pourquoi précisément l'homme, celui qu'Aristote désignait comme le "vivant doué de parole » [zôon logon ekhon], dans la mesure où l'on assortit à sa détermination d'essence l'apanage des latitudes virtuellement infinies que lui prodigue l'usage des moyens finis du langage, demeurera à jamais étranger à lui-même, puisque l'élément où il apprivoise sa propre déshérence en regard de toute origine, bref sa condition d'étranger, ne peut être apprivoisé dans cet élément. L'être est métis.

Cette considération peut paraître abstruse, et je vais tenter de m'en expliquer. D'abord ceci : le fait que nous soyons depuis toujours livrés à l'exercice d'une langue et, de là, qu'il y ait déjà, envers et contre toute espèce de réduction métalinguistique, une pluralité non subsumable de langues, cela ne peut s'exprimer dans les limites et par les ressorts mêmes de cet exercice. Cette impossibilité est le levier, sinon la matrice de l'acte de traduction, le lieu même de son 
assignation. Notre rapport au langage ne peut s'exprimer que dans le langage, mais ce fait ne s'y exprime pas comme tel, il nous oblige à le traduire, à nous y traduire, de sorte que nous sommes toujours sous le coup d'une extradition, du « traduire » comme extradition'. Tout ce qui fait sens est intimement lié à son incommunicabilité. Non pas ce qui " tombe » sous le sens, mais que cela soit, que ce qui fait sens le fasse de telle façon qu'il nous échoit à travers l'exercice d'une langue qui ne trouve sa justification, ou sa raison d'être, ni en elle-même ni en dehors d'elle. Toute tradition, en tant qu'elle ressortit à une interprétation paradigmatique ou canonique des artifices au moyen desquels nous procédons à la mise en discours des données de l'expérience, est fonction d'une extradition originelle, d'un exil de la parole qui s'inscrit dans le sillage d'une trace qu'elle tend à oblitérer et qu'elle ne peut d'autant porter à son actif qu'il lui appartient seulement de creuser son propre passif dans les ressources d'une expression dont l'intelligence est pure transcendance scellée dans son abandon au don de la langue. L'élément de cette transcendance, la mesure de cet abandon est le poème.

J'ai toujours été intrigué, peut-être plus qu'il ne faut, je ne sais trop, par l'affirmation de Wittgenstein à l'article 4.121 de son Tractatus, selon laquelle " ce qui s'exprime dans la langue, nous ne pouvons par elle l'exprimer ${ }^{3}$. Je traduirai volontiers cet énoncé par : que quelque chose soit communicable, cela ne peut se communiquer. Cette proposition est directement solidaire d'une autre qui cheville comme une clef silencieuse l'armature discrète qui jalonne la descente vertigineuse du Tractatus : que [dass] le monde soit, cela même qui fait question, est parfaitement indifférent aux propositions qui expriment " comment " [wie] il est. L'abîme entrouvert par cette question, que d'aucuns estiment dénuée de sens, s'ouvre et se referme aussitôt sur le langage, s'y abolit comme insoluble : que le langage soit ne se laisse pas dire par ce qu'il permet de dire. Ce que nous n'arrivons pas, n'arriverons peut-être jamais à dire, nous serre au plus près; autrement dit, comme Hölderlin le signifiait à Böhlendorf dans sa lettre de décembre 1801, "l'usage libre de ce qui nous est propre est la chose la

2 J'ai posé les jalons de cette analyse dans "Du "traduire" comme extradition ", dans Discours social/Social Discourse, Vol. 5, 3-4, été-automne 1993, pp. 93-98.

${ }^{3}$ Ludwig Wittgenstein, Tractatus logico-philosophicus, traduction, préambule et notes de Gilles-Gaston Granger, Paris, Gallimard, 1993, p. 58. 
plus difficile ». Et si la folie nous guette, ce n'est pas que nous risquons de perdre la raison. Celle-ci n'a jamais rien eu à y voir. Davantage encore, ce qui fait de nous des êtres de raison - on aime à le penser n'est pas du tout rationnel ou raisonnable. La réserve inviolable du silence est le dire lui-même, qui ne peut être dit. Sa pudeur la plus secrète est le vertige qui naît non pas de l'angoisse pour le néant, comme le concevait Heidegger, mais de la sourde étreinte de l'être qui, tel un cercle dont le centre est partout et la circonférence nulle part, pèse d'un poids insensible à l'épiphanie fugitive de la grâce, comme l'impossibilité de mourir, le ressassement éternel de la nuit derrière la nuit.

Nous n'avons accès qu'au " comment"; nous nous butons sans cesse au " pourquoi »- on songe évidemment à la question fameuse de Leibniz, qui a été ruminée ad nauseam par Heidegger : pourquoi y a-t-il quelque chose et non pas rien? -, mais le « quoi », la propriété absolue du communicable, si l'on peut dire, nous demeure inaccessible dans l'élément même où elle se prodigue et nous sollicite comme débiteurs d'une dette que nous n'avons jamais contractée et qui nous oblige d'autant que nous ne pourrons jamais nous en acquitter.

Cet accès nous est interdit, un peu comme l'homme de la campagne dans la parabole de Kafka, Devant la loi, formulant son désir d'approcher le cœur de la loi, demeure interdit, creuse par son attente cette distance infínie qui le rive au seuil d'une porte désespérément ouverte, qui le nargue de sa béance. Le gardien de ce seuil est l'ironie même, puisqu'il est là simplement pour rappeler à l'aspirant qui, au bout de ses forces, s'apprête à mourir, que cette entrée lui était réservée, à lui et à lui seul. L'ironie du gardien de la loi est aussi, mutatis mutandis, l'ironie d'une langue qui peut tout dire sauf en quoi et pourquoi elle le peut : en quoi et pourquoi nous sommes depuis toujours " tombés » dans la langue, et ce de façon autrement singulière que la modalité selon laquelle tel ou tel autre individu donné, donc " nommé ", dans la mesure où il affiche une propriété qui lui permet de se substituer à tout autre individu partageant cette propriété, et vice versa, est dit "tomber" sous le concept. Pour user à nouveau de ce terme de l'héraldique ou de la science du blason qui est en faveur chez les mandarins de la post-modernité, il y a lieu de parler d'une mise en abyme du mortel, donc du sujet parlant, dans l'élément où il peut communiquer quelque chose sous la condition expresse de l'incommunicabilité de cette possibilité même. 
Il ne s'agit pas ici de cultiver quelque disciple de l'arcane, de célébrer quelque chose d'ineffable, mais de marquer de l'intérieur cette frontière abyssale, proprement indéfinie, qui s'ouvre dans l'exercice même d'une langue dite " maternelle ». Je dirai ici que l'écart absolu entre une langue et une autre, celle qui doit traduire par exemple, ne joue pas tant là où l'incompatibilité se veut évidente, comme si on savait déjà mesurer cet écart, mais dans le rapport à l'étranger qui régit secrètement l'impropriété de sa propre langue, qui la révèle comme étrangère à elle-même, comme si le temps surprenait dans ce qui est déjà encodé et "parfait" de quelque façon un état de latence et d'inachèvement qui en appelle à sa rédemption par l'étranger.

Cette condition vaut aussi pour la langue qui est candidate à la traduction, plus précisément pour l'un des multiples états de cette langue intriquée à une facture poétique qui, bien qu'elle se présente comme une construction finie de la langue, sollicite déjà le partage d'une frontière qui n'est absolument pas limitrophe à l'autre langue, mais qui s'instruit dans l'assomption d'un mandat sans origine, en attente d'un possible qu'elle appelle tout en lui demeurant originellement étrangère. Peut-on concevoir une frontière qui lie deux royaumes qui ne sont absolument pas limitrophes?

La notion de frontière, que j'ai introduite dans une précédente étude sans vraiment $m$ 'en expliquer', n'a d'autre résonance que ce partage indéfini d'une décision qui compromet le traducteur, qui l'exile hors des avenues ménagées par la compétence de ce spécimen de locuteur qu'on imagine apte à mobiliser, à tout venant et sans trop s'y attarder, les ressources de sa langue maternelle. Dans sa notion même, une frontière ne renvoie à rien de tangible. Certes se concrétise-t-elle dans une borne, le tracé d'une ligne qui répond à l'arbitraire d'un décret, sinon au relief qui marque une résistance, un point d'arrêt dans la progression du corps nomade. Pour ma part, j'y pressens l'empreinte du temps, un point de passage qui ne se laisse définir ni par la provenance ni par l'arrivée. Même à considérer une frontière dite " naturelle ", celle que dessine un cours d'eau ou le versant d'une montagne, on en vient à l'écriture du temps, qui est facteur d'érosion mais qui draine aussi une multitude d'alluvions où sommeille la fièvre très ancienne des magmas vomis des entrailles du creuset sidéral.

${ }^{4}$ Laurent Lamy, « Pas de deux : le mésocosme de la traduction comme matrice d'une sémantique frontalière ", Méta, vol. $40, \mathrm{~N}^{\circ} 3$, septembre 1995, pp. 461477. 
L'œkoumène, l'espace terrestre habité, plus précisément celui qui permet au vivant de se perpétuer et d'accroître sa complexité, est marqué par l'écriture du temps qui se concentre et se dissémine dans la pluralité des langues où l'espèce humaine prend figure, se construit une mémoire et tente d'apprivoiser la teneur d'un destin, l'ascendant d'une origine dont elle demeure à jamais orpheline.

À cet égard, il est absurde de considérer, comme certains le supposent, qu'il y ait une perte ou une forme d'entropie lorsque la traduction se saisit d'un fragment d'une langue-source pour lui ménager une avenue dans un fragment d'une langue-cible qui, censément, devrait fournir une équivalence en regard d'un signifié, transcendantal ou autre, qui serait l'élément à traduire. Dire, comme je l'affirme, que nous sommes orphelins de toute origine, signifie que le sens, l'épreuve du sensé, l'événement même de la signifiance, ressortit à l'unicité de la forme que révèle l'exercice de la langue dans la langue et non pas à l'unité d'un contenu qui serait extérieur à cet exercice qui dans son essence même est marqué par un état originel de dissémination, par le frayage diasporique des langues qui honorent par leur disparité notre commun partage de l'œkoumène. L'amplitude de la différence entre les divers idiomes où s'inscrit la trace de cette dissémination répond au tracé indéfini de cette frontière que la traduction franchit sans jamais pouvoir l'effacer, ne laissant plutôt de l'approfondir en ampleur et en intensité dans le tranchant de la décision, dans la coupure décisoire qui l'exile à l'interface de diverses nébuleuses sémantiques, trouvant alors sa condition de possibilité, cela est bien connu, dans son impossibilité même.

L'affirmation de Wittgenstein que j'ai portée à votre attention plus haut n'allait sans doute pas aussi loin, encore qu'il faille ici s'armer de nuances. Lorsque Wittgenstein affirme que «ce qui s'exprime dans la langue, nous ne pouvons par elle l'exprimer ", il réfère entre autres à l'asymétrie entre le dire et le montrer. À l'article 4.1212 du Tractatus, il stipule que " ce qui peut être montré ne peut être dit "; c'est-à-dire que nous ne pouvons nous exprimer ou formuler quoi que ce soit de sensé quant au fait que tel ou tel autre état de choses prévale et qu'il puisse seulement être montré par la forme logique de la proposition. L'abîme entre l'exhibition d'un état de choses, le régime de l'ostension, et la possibilité de dire ce en quoi et pourquoi il en est ainsi est proprement inscrutable. La catharsis très rigoureuse à laquelle Wittgenstein soumet l'exercice de la pensée dans le Tractatus consiste à "délimiter l'impensable de l'intérieur par le moyen du pensable » 
(4.114). La clef du Tractatus, faut-il le préciser, ne se trouve cependant pas dans ce qu'il délimite comme le domaine du pensable, les paramètres du dicible étant alors circonscrits de l'intérieur, par l'exhibition des états de choses composant la figure du monde à l'aide des propriétés affichées par la forme logique de la proposition. Cette clef se trouve plutôt dans ce qui n'y est pas exprimé, soit l'éthique. Dans une lettre bien connue que Wittgenstein adressait en octobre 1919 à Ludwig von Ficker, le penseur viennois y allait de cette mise au point :

Mon travail consiste en deux parties : celle qui est ici présentée [dans le Tractatus] plus tout ce que je n'ai pas écrit. Et c'est précisément cette seconde partie qui est la partie importante. Mon livre trace, pour ainsi dire, de l'intérieur, les frontières de la sphère de l'éthique, et je suis convaincu que c'est là la SEULE façon rigoureuse de tracer ces frontières. J'ai fait en sorte dans mon livre de tout mettre bien à sa place en gardant le silence là-dessus. ${ }^{5}$

J'ai tenu à rappeler cette exigence extrême à laquelle Wittgenstein a plié son propos, parce que j'estime qu'il y a une analogie et même une certaine affinité qui se dessine entre cette façon de tracer de l'intérieur les frontières de l'éthique et la frontière que la traduction doit franchir sans jamais l'abolir, puisqu'elle s'inscrit dans la trace d'une dissémination qui précède toute inscription d'un signifié auquel elle devrait censément se rapporter pour s'accomplir comme traduction. Antérieurement à toute conception d'un langage devant communiquer quelque chose, il y a la pluralité des langues dont l'irréductibilité détermine un rapport au temps qui ne peut " tomber " sous le concept. Ce rapport est violent, ou plutôt soumis à une violence tacite puisqu'il est offusqué dans le type de traduction qui tient son droit de cité de l'oblitération de sa propre trace, celle du temps qui opère dans la traduction. Si l'on s'en fie à une notion idéale du résultat auquel devrait aboutir une traduction, celle-ci tiendrait sa " félicité » de son propre effacement. Mais pareille dénégation, qui la confine à une fonction ancillaire, est le signe contraire de son exigence la plus haute, c'est-àdire que le résidu ou la trace de la langue qui ne se laisse pas indexer par la blanche écriture du concept commande l'éthique même de la traduction qui, avant d'être la mesure d'une compétence ou d'une

${ }^{5}$ Cité et traduit par Grahame Lock, dans Wittgenstein. Philosophie, logique, thérapeutique, traduit par Jeanne Balibar, Philippe Mangeot et l'auteur, Paris, PUF, 1992, p. 59. 
maîtrise technique, est, sous la condition expresse de son impossibilité, la révélation même de la communicabilité, cela précisément qui ne se communique pas.

Maintenant, comme nous le rappelle Giorgio Agamben dans une étude qu'il a consacrée au travail de Jacques Derrida, la thèse de Wittgenstein selon laquelle « ce qui s'exprime dans la langue, nous ne pouvons par elle l'exprimer ", mène à son ultime conséquence un paradoxe entrevu déjà par Gottlob Frege dans un article de 1892, intitulé "Objet et concept ». Ce paradoxe, qui allait ébranler l'édifice de la logique formelle, s'énonçait alors comme suit : " le concept cheval n'est pas un concept $\%$. Comme le note Agamben, il y a ici matière à paradoxe dans « le fait que chaque fois que nous nommons un concept (au lieu de l'utiliser comme prédicat dans une proposition) il cesse de fonctionner comme concept et se présente comme objet "; en fait, le paradoxe de Frege est "la conséquence d'un principe plus général qui peut s'énoncer ainsi : un mot ne peut dénoter quelque chose et, en même temps, dénoter qu'il le dénote $~^{6}$. C'est ce même paradoxe que Lewis Carroll, alias Charles Lutwidge Dodgson, dont on sait qu'il fut un logicien chevronné, à tout le moins astucieux même s'il en était encore à ressasser les ruines de l'Organon aristotélicien, nous invitait à considérer dans la boutade du Cavalier blanc dans $\dot{A}$ travers le miroir : "le nom du nom n'est pas le nom ». À vrai dire, et quoique Agamben ne le mentionne pas, on peut remonter à Leibniz, qui explique l'identité comme une relation entre signes, et non entre un objet nommé et luimême. ${ }^{7}$ Et sans doute pourrait-on retracer dans son état naissant tout le réseau d'apories suscitées par le problème de l'identité dans les paradoxes qu'inventaient et s'efforçaient de dénouer les logiciens médiévaux, qui renouvelaient en empruntant de multiples ramifications les disputes d'école initiées chez Platon et Aristote, chez les Stoïciens et les Mégariques notamment, et qui tiennent pour l'essentiel au

"Giorgio Agamben, "Pardès. L'écriture de la puissance ", dans Revue philosophique de la France et de l'étranger, $\mathrm{N}^{\circ} 2$, avril-juin 1990, pp. 139140.

${ }^{7}$ C'est ce que rappelle W. V. Quine dans son ouvrage Quiddités. Dictionnaire philosophique par intermittence, trad. par Dominique Goy-Blanquet et Thierry Marchaisse, Paris, Seuil, 1992, p. 258. 
différend insoluble qui confronte les zélateurs respectifs du nominalisme et du réalisme. ${ }^{8}$ Le « nom de la rose ", en fin de compte. ${ }^{9}$

Deux remarques s'imposent ici. D'abord, dans la mesure où la traduction n'a pas seulement à rencontrer l'index qui lie le signifiant au signifié, par quoi on dénote quelque chose sous condition de préserver l'univocité de la référence, mais doit aussi s'acquitter de la livraison d'un signifiant qui ne recouvre pas nécessairement le spectre sémantique de l'élément à traduire, elle ne peut faire l'économie de cette disjonction interne qui fait matière à paradoxe et qui, pour les fins de la logique, doit être sanctionnée, si un énoncé doit être réputé valide ou simplement doué de sens. La traduction doit aussi s'occuper du " nom du nom », de l'élément infra-sémantique où le concept est coulé, parfois floué. L'assignation de cet élément est proprement a-topique, c'est-à-dire qu'il échappe au clivage entre sens et référence. Les attentes de la traduction, en cela même qu'elles ne sauraient être comblées, et c'est là la mesure de son abandon au don de la langue, se heurtent à cette strate profonde de la langue où l'épaisseur du temps est encodée et dont la gestation l'accule en deçà de la dotation même de la semiosis comme processus ininterrompu de transformation appelé à se cristalliser dans un dispositif agréant l'itération univoque d'un signifié à travers la diversité de ses occurrences. Bref, ce qui est dit dépend toujours de la façon de le dire et, qui plus est, cette façon de dire est la marque même de ce qui est à traduire et qui s'impose comme impossible à traduire. C'est le cas notamment pour le matériau poétique dont la facture répond à l'exercice de la langue dans la langue, un exercice qui, par conséquent, est essentiellement " performatif ", c'està-dire sui-référentiel dans le sens où on l'entend dans la pragmatique des actes de langage. Pour reprendre la formule utilisée plus haut, je dirais que le poème "dénote qu'il dénote " bien davantage qu'il ne dénote quelque chose. Si le poème est toujours au creuset, en attente d'une lecture qui va le délivrer de ce qui en lui est absolument " parfait », achevé, consommé, c'est qu'il porte la cicatrice du temps, la marque de sa propre finitude, et c'est pourquoi il se prête à une interprétation infinie.

\footnotetext{
${ }^{8}$ Pour une généalogie de ces disputes, voir Alain de Libera, La querelle des universaux. De Platon à la fin du Moyen Âge, Paris, Seuil, 1996.

${ }^{9}$ Sur l'influence des théories médiévales de la signification dans le roman d'Umberto Eco, voir l'ouvrage de Theresa Coletti, Naming the Rose. Eco, Medieval Signs, and Mociern Theory, lthaca et Londres, Cornell University Press, 1988.
} 
Aussi la traduction ne peut-elle faire l'économie de ce qui est exclu par une décision initiale, une espèce de décret implicite ou tacite, dans le cadre de la logique. Pour le traducteur, la décision est toujours ouverte, l'affaire, toujours en suspens, pendante. Par contre, l'affaire de la logique, qui est un " enfer " comme aimait à le rappeler Bertrand Russell, est précisément de surseoir à cet abîme, s'arracher à son empire. Évidemment, je simplifie à l'extrême. ${ }^{10}$ Quoi qu'il en soit, la forme logique de la proposition requiert la saturation sémantique de tout énoncé. La gestion du concept doit non seulement présumer de l'univocité du renvoi de signifiant à signifié, et ainsi répudier le tiers [tertium non datur], conjurer le sort de l'équivoque nourrie par le « nom du nom », mais encore doit-elle se départir du messager pour s'occuper exclusivement du message. Le témoin est tenu à forclusion, il est toujours jugé par contumace, puisque le sujet de la proposition logique n'est susceptible de " tomber " sous le concept que si tout autre individu affichant les mêmes propriétés peut lui être substitué. La traduction, elle, est compromise par le témoin - un témoin qui se trahit dans la langue, ou qui y trouve sa rédemption, une espèce de sursis. Car l'exercice de la langue dans la langue trahit toujours un rapport à la mort, le savoir d'un non-savoir, l'ébauche d'une survie. Dans une allocution prononcée à Brême, le poète juif d'origine roumaine et d'expression allemande Paul Celan, dont on sait qu'il fut un survivant devant survivre à sa propre survie et qu'il n'a pu ultimement y survivre, fait allusion, comme le note Maurice Blanchot, à « ce qu'a pu signifier pour lui — et par lui pour nous - la possibilité qui ne lui a pas été retirée d'écrire des poèmes dans cette langue à travers laquelle la mort vint sur lui, sur ses proches, sur les millions de Juifs et de non-Juifs, événement sans réponse. " Je cite ce passage dans la traduction de Blanchot, tirée de sa très belle étude intitulée Le dernier à parler :

Accessible, proche et non perdue, restait, au milieu de tout ce qu'il avait fallu perdre, cette seule chose : la langue. Elle, la langue, restait non perdue, oui, en dépit de tout. Mais il lui fallut alors passer par ses propres absences de réponse, passer par un terrible mutisme, passer par les mille épaisses ténèbres d'une parole meurtrière. Elle est passée sans se donner de mot pour ce qui avait eu lieu. Mais elle

\footnotetext{
${ }^{10}$ Pour une perspective vraiment novatrice sur le développement et les limites de la logique, voir le dernier ouvrage de Keith Devlin, Goodbye, Descartes. The End of Logic and the Search for a New Cosmology of the Mind, New York, John Wiley \& Sons, 1997.
} 
passa par ce lieu de l'Événement. Passa et put à nouveau revenir au jour, enrichie de tout cela. C'est dans ce langage que, durant ces années et les années d'après, j'ai essayé d'écrire des poèmes : pour parler, pour m'orienter et apprendre où je me trouvais et où il me fallait aller pour que quelque réalité s'ébauchât pour moi. C'était, nous le voyons, événement, mouvement, cheminement. C'était l'essai pour gagner une direction. ${ }^{11}$

Il s'agissait de passer la frontière intérieure de la langue, ne pouvant qu'habiter ce reste sans reste, ce "SINGBARER REST ", ce " résidu chantable ", alors que le réel n'offrait plus aucune espèce de refuge, nulle alvéole, nulle anfractuosité où couver cette blessure insuturable, cette déchirure inscrite dans une langue qui est celle de la mort : " der Tod ist ein Meister aus Deutschland - la mort est un maitre venu d'Allemagne ${ }^{12}$.

Mais la traduction n'est-elle pas aussi, de quelque façon, extradée vers cette frontière intérieure de la langue où les idiomes communiquent d'abord leur irréductibilité, leur résistance à toute forme d'indexation au profit d'un tuteur transcendantal balisant l'espace logique où s'inscrivent les énoncés faisant profession de vérité? Opérateur apatride, la traduction est exilée dans l'interrègne où la langue s'apprivoise dans son étrangeté foncière, le " don de la langue», la Gabe der Sprache au sens où Walter Benjamin s'en est enquis, ne pouvant alors se révéler qu'à la mesure d'un abandon, d'une Aufgabe qui seule peut assumer le fait que l'être-langue de la langue est déjà inscrit, depuis toujours, dans l'espace ouvert par l'appel de l'autre.

C'est pourquoi j'ai pu suggérer plus haut que la zone frontalière où la traduction se voit en quelque sorte extradée, l'entredeux-langues, n'est pas sans rappeler l'inscrutabilité à laquelle est

"Maurice Blanchot, Le dernier à parler, Montpellier, Fata Morgana, 1984, pp. 43-45.

${ }^{12}$ Cette phrase est répétée quatre fois dans diverses constructions du grand poème de Celan, «Todesfuge », rédigé en 1944 et paru d'abord en roumain en 1947, dans la traduction de son ami Petre Solomon, et en allemand en 1952, dans son recueil Mohn und Gedächtnis (Pavot et mémoire), trad. par Valérie Briet, Paris, Christian Bourgois, 1987; voir P. Celan, Gesammelte Werke, I, éd. par Beda Allemann et Stefan Reichert, avec Rolf Bücher, Frankfurt am Main, Suhrkamp, 1983, p. 141. 
acculée la quête d'un fondement pour l'éthique ${ }^{13}$, qui renvoie à l'abîme du langage, à la rigueur d'un commandement dont la source transcende la possibilité même de se conformer à un impératif catégorique.

Maintenant, j'en viens à la seconde remarque que j'annonçais plus haut, en évoquant la thèse de Wittgenstein dans le sillage du paradoxe de Frege et des diverses apories liées à la clause selon laquelle un nom ne peut dénoter le fait qu'il dénote sans enrayer la procédure rationnelle qui permet de conserver l'identité du signifié visé ou véhiculé par le signifiant. Il s'agit du nom propre dont Jacques Derrida a pu montrer ${ }^{14}$ qu'il a partie liẻe avec l'irréductibilité de l'élément au sein duquel la traduction doit se frayer un passage, c'est-àdire négocier avec le tropisme pur de la langue pour ménager ses propres avenues. Ce qu'elle ne peut faire qu'en se butant à l'impropriété même de ce qui lui est le plus propre, puisque le commerce avec l'étranger découvre l'étrangeté même de la langue propre, celle qui est dite " maternelle ". Ceci dit, il est clair que nous n'avons pas accès à ce que je viens de désigner comme le tropisme pur de la langue, en référence à la notion pas du tout évidente de reine Sprache mise de l'avant par Walter Benjamin dans son texte fameux sur la « tâche » du traducteur. Que cet accès nous soit interdit n'est pas la marque d'un défaut, d'une carence, d'une chute, d'une dégénérescence en regard d'une faculté de langage idyllique,

${ }^{13}$ Lors d'entretiens avec les membres du Cercle de Vienne tenus chez Moritz Schlick le 30 décembre 1929 et rapportés par Friedrich Waismann, Wittgenstein marquait ce point crucial (Manifeste du Cercle de Vienne et autres écrits, publié sous la direction de Antonia Soulez, trad. par Barbara Cassin, Anne Guitard, Jan Sebestik et Antonia Soulez, Paris, PUF, 1985, pp. 250-251) : « Il y a en l'homme la pulsion de s'élancer contre les frontières du langage. Pensez par exemple à l'étonnement dû au fait que quelque chose existe. Cet étonnement ne peut pas s'exprimer sous la forme d'une question de même qu'il ne comporte absolument pas de réponse. Tout ce que nous pourrions dire ne peut être a priori que non-sens. Il n'empêche que nous nous élançons contre les frontières du langage. Kierkegaard lui aussi a bien vu cet élancement et le décrit dans des termes tout à fait semblables (comme manière de s'élancer contre le paradoxe). S'élancer contre les frontières du langage, c'est là l'éthique. »)

${ }^{14}$ Voir J. Derrida, "Des tours de Babel », Psyché. Inventions de l'autre, Paris, Galilée, 1987, pp. 208-209; "Fors", préface à Le verbier de l'homme aux loups (Anasémies $I$, de Nicolas Abraham et Maria Torok, Paris, AubierFlammarion, 1976, pp. 71-72. 
paradisiaque, dont l'espèce humaine aurait joui dans un lointain jadis. C'est que le langage, loin de se vouloir un dispositif de communication ou un moyen d'expression dont un agent locuteur saurait disposer à son gré en fonction de sa compétence linguistique, est une forme de vie, un milieu ou un élément dans lequel le sujet parlant évolue, se perd et se retrouve sans jamais pouvoir s'en abstraire. L'homme est sujet au langage, lui est assujetti bien davantage qu'il n'en est le sujet. Or c'est là précisément où le sujet est confronté à ce sur quoi il ne peut absolument rien, la réalité même de la langue étrangère, que se révèle l'étrangeté du rapport à la langue qui lui est propre, l'être-langue de la langue, cela même qui est visé par la traduction ${ }^{15}$ et qui, tel que Benjamin s'est efforcé de nous le faire entendre, s'attache davantage au mode de viser [die Art des Meinens] qu'à la chose visée [das Gemeinte] ${ }^{16}$.

Dans l'une des épigraphes de son ouvrage intitulé La parole donnée, et qui concerne de très près la question qui nous occupe ici, Alexander Garcia Düttmann nous reporte à l'affirmation de Benjamin selon laquelle "Rien ne lie mieux un homme au langage que son nom ${ }^{17}$. En marquant ce lien, il ne s'agit évidemment pas de ratifier une manière assez convenue de narcissisme. Le nom propre, dont on sait qu'il peut facilement faire l'enjeu chez maints écrivains d'une incorporation cryptique, marque la langue par son extra-territorialité, par son caractère indéclinable, dans la mesure justement où il est associé à la déclinaison d'une identité qui n'a pas de contenu. Le nom propre appartient à la langue sans lui appartenir, il est exclu de son fonctionnement en tant qu'elle doit communiquer quelque chose. Le nom propre ne se laisse pas traduire. Son signifié, s'il en est, est indissociable de son substrat phonique, de sa forme graphique et de la

${ }^{15}$ Ce qui est visé par la traduction, comme l'écrit Jacques Derrida (Psyché, p. 232), c'est « la langue même comme événement babélien, une langue qui n'est pas la langue universelle au sens leibnizien, une langue qui n'est pas davantage la langue naturelle que chacune reste de son côté, c'est l'être-langue de la langue, la langue ou le langage en tant que tels, cette unité sans aucune identité à soi qui fait qu'il y a des langues, et que ce sont des langues. "

${ }^{16}$ Walter Benjamin, « L'abandon du traducteur. Prolégomènes à la traduction des "Tableaux parisiens" de Charles Baudelaire ", traduction et notes de Laurent Lamy et Alexis Nouss, dans TTR, Vol. X, N² 2, 1997, p. 19.

${ }^{17}$ Alexander Garcia Düttmann, La parole donnée. Mémoire et promesse, Paris, Galilée, 1989, p. 11. 
non-substitutivité de son référent. Bref, le nom propre ne « tombe » pas sous le concept.

Le nom propre ne signifie rien, ne communique rien. Il s'inscrit dans l'espace ouvert par l'appel de l'autre, un peu comme la mort que tout un chacun sait nommer sans pouvoir en fournir le référent. Le nom propre est la marque d'un endettement mutuel, inflationniste, entre deux mortels. Le nom propre est intriqué à la trace d'un passé qui ne fut jamais présent, la trace d'un passé comme futur antérieur, l'à-venir de la mort en guise d'à-Dieu inscrit, tel un point de fuite, dans l'abstraction inouĩe du visage humain, dans cette déchirure pétrie de chair et de souffle qui déserte les horizons du monde comme pure effraction d'une trace sans origine, datation d'un événement qui passe en étant déjà passé, d'un individu qui s'en va à la mort, qui endeuille déjà le survivant, qui l'endette au-delà de sa capacité d'assomption, qui creuse son passif à l'infini. ${ }^{18}$

L'être humain est le mortel. Le mortel ne l'est que parce qu'un nom propre lui a été octroyé, avant même qu'il puisse s'identifier à lui et par lui. L'oblitération du nom propre signifie l'impossibilité de mourir, de laisser une trace et d'endeuiller le survivant, de l'endetter au-delà de la mort, la mort qui n'est pas, mais qui vient toujours. Les nazis savaient cela, puisque pour éradiquer l'homme juif, pour extirper la judéité même de l'homme hors du règne de l'être, il fallait d'abord le dépouiller de son nom propre, lui affubler un matricule. Mais la disparition des corps immatriculés, qui n'est pas la mort mais une simple disparition, ne pouvait être consommée qu'en oblitérant la trace de cette disparition. Il faut faire en sorte que ce qui disparaît n'existe pas, qu'il n'ait même jamais existé - faire en sorte qu'il lui soit même impossible de mourir. Pour qu'une telle logique aboutisse, il faut, comme l'écrit Jean-François Lyotard dans Le différend, non seulement anéantir le nom propre individuel, mais

[...] il faut tuer aussi le nom collectif (juif), de façon qu'aucun nous porteur de ce nom ne reste qui puisse reprendre en lui et pérenniser la

\footnotetext{
${ }^{18}$ En m'exprimant de telle façon, je ne peux que marquer ma dette à l'endroit de la pensée d'Emmanuel Lévinas, sans laquelle je n'aurais pu m'aventurer sur ce terrain éminemment difficile, pour ainsi dire impraticable; voir Humanisme de l'autre homme, Montpellier, Fata Morgana, 1972, et Autrement qu'être ou au-delà de l'essence, La Haye, Martinus Nijhoff, 1974.
} 
mort du déporté. Il faut donc tuer cette mort, et c'est ce qui est pire que la mort. Car si la mort peut être anéantie, c'est qu'il n'y a rien à faire mourir. Pas même le nom de Juif. ${ }^{19}$

Le nom propre signifie aussi qu'il n'y a pas la langue mais des langues qui fonctionnent en excluant de leur fonctionnement, en marquant d'une impropriété majeure ce qui leur est le plus propre et qui ne se laisse pas traduire. Le nom propre frappe de caducité la pertinence du débat qui remonte au moins au Cratyle de Platon, en ce qui a trait au caractère conventionnel ou motivé de telle ou telle autre dénomination, ou de tel ou tel autre élément significatif du langage. Que je m'appelle Laurent Lamy n'est absolument pas rentable du point de vue cognitif, mais ne nuit pas non plus au fonctionnement de la langue où ce nom peut être mobilisé comme label pour décrire l'individu que je suis en lui greffant une série de prédicats qui me permettent de « tomber » sous le concept, par exemple de m'identifier comme individu du genre "homme" ou de constater que nous sommes là en présence d'un mortel. Mais le nom propre n'appartient pas comme tel à ce fonctionnement.

La seule existence d'un nom propre, qui demeure intraduisible en cela même qu'il n'appartient pas au fonctionnement de la langue comme véhicule de communication, révèle l'hétérogénéité de la langue en regard de sa propre fonction. L'impossibilité de traduire le nom propre est l'emblème silencieux de la traduction originaire qui commande toutes les traductions, qui les situe toujours à la frontière d'une communicabilité qui permet de communiquer parce qu'ellemême s'avère incommunicable. Que le tropisme même de la langue, telle ou telle autre tournure ne puisse migrer vers l'autre langue qu'au prix d'une métamorphose qui l'affranchit, tout en les révélant, des contraintes formelles liées à son inscription comme expression douée de sens, révèle une frontière qui ne peut qu'être creusée - Kafka parlait dans l'un des ses Carnets de creuser la " fosse de Babel 》. Cette frontière sans origine où est condensée toute la stratification des états de la langue traversée par l'écriture du temps, est mise à l'épreuve dans le mouvement d'exode auquel se destine la traduction, qui ne laisse ainsi de l'approfondir dans l'ouverture d'un rapport qui ne peut se refermer sur lui-même, car son initiative n'appartient ni à l'un ni à l'autre des termes mis en relation, pas plus qu'il ne les détermine comme termes de cette relation. Une langue n'existe pas en vertu de ce

${ }^{19}$ Jean-François Lyotard, Le différend, Paris, Minuit, 1983, p. 150. 
rapport, mais elle se révèle à elle-même dans ce rapport. Nous ne pouvons produire le fondement de ce rapport, que j'ai associé à l'arbitraire d'un décret qui en fin de compte demeure inscrutable, parce que nous nous situons toujours dans la langue, comme s'il nous était donné de parcourir un envers sans endroit.

La traduction ne peut que creuser cette frontière qui délimite un no man's land, cet espace sans dimension, complètement dés-astré, pour reprendre ici un terme de Maurice Blanchot, que dessine notre intrication originaire à ce qui dans la langue ne se laisse pas circonscrire ou identifier et qui livre le traducteur à cet abandon premier qui le confronte précisément au don de la langue, cela même qui ne se traduit pas. Pareil abandon l'assigne en retour comme un no land's man, œuvrant dans cet envers sans endroit qui révèle l'êtrelangue de la langue, la composante chorale et diasporique de sa matrice, son état originel de dissémination qui extrade le sujet parlant en deçà de l'origine, l'acculant à l'impossibilité même de recouvrer ce déjà-là, ce tropisme pur de la signifiance sur lequel l'élément de la pensée cultivant des certitudes retardera à jamais, jamais ne pourra se dessaisir du retard constitutif de son exercice.

La traduction ne peut s'abstraire de ce qui s'impose à elle comme intraduisible et qui l'exige et la sollicite comme tel. Dans la mesure où la traduction est toujours sollicitée par ce qui l'exige et lui demeure foncièrement étranger, c'est-à-dire peut aussi bien s'en dispenser, son mandat porte la marque du temps, celle de la mort, le savoir d'un non-savoir, l'endettement indéfini d'un fragment de la langue qui migre vers un autre fragment de la langue, comme des épaves portées par un océan de douleur, les cendres des multitudes, l'épreuve du temps qui octroie et qui retranche, le deuil impossible d'une mémoire arrachée à sa propre trace, l'étreinte du vide qui se creuse dans l'absence absolue du témoin, dans la postérité inassumable d'Auschwitz, par exemple.

La métonymie, la substitution de la partie pour le tout, ou d'une partie à une autre, serait le trope canonique de la traduction basée sur le régime de l'équivalence ou la correspondance univoque du signifié au signifiant dans la translation d'une langue-source vers une langue-cible. Selon cette conception, les mots ou les syntagmes de chaque langue sont perçus comme des éléments ou des parties d'un tout transcendantal qui serait la signification ou le sens qui doit être traduit. À la limite, on peut y aller d'un raisonnement bâtard en nous prévalant 
ici des termes d'une analyse célèbre de Noam Chomsky : si la structure profonde correspond à ce qui est susceptible d'être partagé par n'importe quel locuteur de n'importe quelle langue donnée, donc si cette structure profonde exprime le sens et que c'est le sens qu'il faut traduire lorsqu'il y a matière à traduction, alors le traducteur ne traduit rien.

Aucune signification, serait-elle de portée universelle, n'est vierge de son inscription dans le corps indigène d'une langue.

L'univocité de la référence visée par un acte de langage n'est, si l'on peut dire, que le "précipité " de la polysémie originelle affectant le renvoi de signifiant à signifié et, a fortiori, le spectre virtuellement infini des relations générées par l'usage non saturé du tropisme de la langue.

L'effet de dissémination qui est oblitéré par la fonction régulatrice du concept assigne l'acte de traduction à son lieu propre, qui est frontalier et extra-territorial, dans la mesure où il lui incombe de négocier avec le frayage de traces insoupçonnées, parfois fortement métissées, qui trahissent la condition plurale, post-babélienne, du ressourcement idiomatique mobilisé dans l'intention de signifier quelque chose. Il en ressort que l'acte de traduction ne peut désormais être relégué à une fonction ancillaire en regard d'un signifié, d'un concept ou d'un tuteur transcendantal censé commander la translation idyllique entre une langue-source et une langue-cible.

La traduction est par essence "performative », c'est-à-dire qu'elle produit du sens. Aussi fait-elle brèche vers une dimension d'universalité qui n'est pas d'abord subordonnée à l'unité du concept, mais se ressource et trouve son épanouissement dans l'unicité de la forme qu'elle délivre, suivant la métaphore consacrée par Walter Benjamin, à la faveur d'un travail d'obstétrique qui révèle l'être-langue de la langue dans sa pure "haeccéité ", pour user ici d'une notion introduite par le philosophe médiéval Jean Duns Scot. ${ }^{20}$ Dans ce cas, il

${ }^{20}$ Chez Jean Duns Scot (1266-1308), en effet, l'individuation du singulier est élevée au niveau de l'essence qui se définit alors comme "l'ultime actualité de la forme », une pure " haeccéité », qu'on peut traduire par " ce-là », " l'étant que voici » (le vocable latin haecceitas, forgé ad hoc par Duns Scot, est substantivé à partir de l'adjectif et pronom démonstratif haec). L'haecceitas dénote l'actualité première et ultime du singulier, l'unicité de sa manifestation où s'accuse la marque propre de l'individualité qui assure la prégnance de la 
ne lui appartient pas de communiquer quelque chose, mais de révéler l'essence même de la communicabilité. C'est la thèse, plutôt hétérodoxe, qui est formulée par Benjamin dans son essai de 1916, Sur le langage en général et sur la langue des hommes. Il en appelle en effet à une conception de la langue qui " ne connaît aucun moyen, aucun objet ni aucun destinataire de la communication ", s'agissant alors d'accéder au nom, à ce " par quoi plus rien ne se communique, et en quoi la langue se communique elle-même absolument. [...] Il n'y $a$ pas de contenu de la langue; en tant que communication, la langue communique un être spirituel, c'est-à-dire une communicabilité pure et simple $\aleph^{21}$.

C'est ce leitmotiv étonnant, du moins assez déroutant, qui va guider le propos de Benjamin lorsqu'il aborde le problème de la traduction, qui pour lui trouve son fondement dans la couche la plus profonde du langage. Le « don de la langue » [Gabe der Sprache] est attention soutenue, originellement incirconscrite, au langage même des choses, qui en appelle à l'abandon du traducteur, à son Aufgabe. Cette " tâche " ou cet abandon au langage des choses encore muettes, encore celées dans la mélancolie de leur « mutité " [Stummheit] — je songe ici

forme dans l'exclusive de toute médiation métaphorique ou métonymique (e.g. la partie pour le tout). L'unicité de la manifestation du singulier précède son inscription sous la juridiction de l'universel. C'est le cas, à mon sens, de toute langue, qui serait un cas d'espèce, mais, en l'occurrence, une espèce qui ne " tombe » pas sous le concept, l'espèce d'aucun genre. Je rappelle que pour Benjamin « Übersetzung ist ein Form », et que pour Wittgenstein l'exercice du langage, loin d'être confiné à une simple fonction instrumentale, constitue en soi une "forme de vie " [Lebensform], et, enfin, que Benjamin rattache le travail de la traduction à l'idée d'une " survie » [Überleben], ménageant l'aval de quelque chose de vivant qui aurait une vie devant soi, et ce malgré soi, une vie hors de soi qu'elle appelle à son insu, qu'elle commande sans la solliciter - le passage de ce qui jouit d'une vie propre vers un seuil qui annonce une autre vie qui a aussi sa vie propre [Fortleben].

${ }^{21}$ Ces deux passages sont cités et traduits dans l'étude de G. Agamben, " Langue et histoire. Catégories historiques et catégories linguistiques dans la pensée de Benjamin ", trad. par Yves Hersant, dans Walter Benjamin et Paris, Colloque international, 27-29 juin 1983, éd. par Heinz Wismann, Paris, Cerf, 1986, pp. 796-797; j'ai cependant restitué l'italique dans la dernière phrase citée, conformément au texte original de Benjamin. Voir aussi la traduction de Maurice de Gandillac, "Sur le langage en général et sur le langage humain », W. Benjamin, Euvres I: Mythe et violence, Paris, Denoël, 1971, pp. 79-98. 
à l'extraordinaire connaissance des individus du monde végétal chez Paul Celan, à cette " science des noms » nourrie par une empathie qui transcende évidemment la simple "curiosité " taxonomique ${ }^{22}$, se destine donc à ce que Benjamin désigne comme l'Empfängnis des Namenlosen im Namen, c'est-à-dire à la " conception dans le nom de ce qui est sans nom ". Nommément, c'est la tâche que Dieu aurait impartie à l'homme dans le mythe biblique de la Création. Laisser vibrer dans le nom ce qui n'a pas de nom, c'est précisément là la tâche $\mathrm{du}$ traducteur. Les langues humaines, vernaculaires aussi bien que véhiculaires, sont dérivées de ce rapport originel qui engage la traduction et lui confère son mandat: franchir l'abîme qui sépare le nom et la chose, qui est la conséquence de la chute dans un langage référentiel qui départage la chose nommée et les propriétés abstraites qui lui permettent de "tomber" sous le concept, bref de projeter $\grave{a}$ travers le nom, d'y re-présenter un contenu extérieur à la chose et qui la définirait dans son essence, indépendamment de la langue où se communique cette essence. La tentative de Benjamin pour percer à jour l'essence du langage dans sa pure teneur spirituelle, loin de se cantonner dans une espèce de nostalgie pour une langue " adamique ", n'est autre que l'effort pour mettre en échec le processus diffus de réification qui s'est progressivement infiltré dans la communication entre les hommes et dont l'empire s'étend à l'ensemble des relations que l'être humain entretient avec toutes les créatures peuplant l'œkoumène. Dans ce cas, la problématique de la traduction n'est pas seulement concernée par la disparité des idiomes qui sont l'apanage exclusif d'un locuteur humain, mais embrasse aussi, selon Benjamin, la spiritualité latente des êtres de tous les règnes, dont l'essence se communique dans l'harmonie virtuelle qui se découvre dans l'amplitude de la différence prospectée par la traduction dans sa migration à travers la diversité des microcosmes langagiers où l'homme fait l'apprentissage de sa propre condition comme symbole vivant du royaume créaturial. Point crucial ici, l' "essence spirituelle" de la chose se communique dans le langage et non pas à travers lui. Cette distinction est en effet la pierre de touche des thèses développées par

${ }^{22}$ En fait, comme nous l'apprend John Felstiner dans le remarquable essai biographique qu'il nous a livré, Paul Celan. Poet, Survivor, Jew, New Haven et Londres, Yale University Press, 1995, p. 96, la bibliothèque de Celan regorgeait d'ouvrages consacrés à la zoologie, la géologie, la minéralogie, la cristallographie, la physique, l'anatomie, l'ornithologie, la botanique évidemment et, tout spécialement, aux roses. 
Benjamin dans son essai de 1916, Sur le langage en général et sur la langue des hommes. Comme le précise Alexander Garcia Düttmann,

[...] dans la mesure où la chose doit se communiquer, où la communication lui appartient, son essence spirituelle est une "essence langagière". La traduction est l'effet de l' "essence langagière" de la chose et, par conséquent, de sa spiritualité. Autrement dit : l'esprit de la chose - ce qui rend possible toute expérience, la "communicabilité" même - commande sa traduction. Mais le langage n'est pas simplement l'expression d'un "contenu" quelconque, d'un "contenu" extérieur à la communication ou à l'énonciation: il est plutôt l'expression de ce qui ne se dit jamais. Ce qui ne se dit jamais est le "dire" comme condition de possibilité du "dit". Par "contenu spirituel", il faut entendre la spiritualité du langage, non un langage spirituel : "Que communique le langage? Il communique l'essence spirituelle correspondante. Il est fondamental de savoir que cette essence spirituelle se communique dans le langage et non par lui". ${ }^{23}$

Deux remarques, pour conclure. D'abord, cette analyse marque une nette proximité avec ce que Wittgenstein donnait à entendre plus haut, dans la mesure où la couche la plus profonde du langage, celle qui sollicite la traduction et son abandon à l'être-langue de la langue, ressortit à l'expression de ce qui ne se dit jamais. L'extrême minutie, la précision quasi chirurgicale qui caractérise chez Wittgenstein l'examen des rapports entre l'expression la plus prosaïque d'un état de choses et l'effet " performatif » qu'entraîne son usage par un locuteur dans une situation donnée, davantage qu'un souci de transparence analytique, dénote plutôt une déférence à la profondeur et à la complexité du paysage qui se découvre dans sa simplicité même sous la surface des choses qui nous apparaissent les plus familières. ${ }^{24} \grave{A}$ cet égard, je songe à la remarque de Paul Celan, dans son allocution de Brême, lorsqu'il

${ }^{23}$ La parole donnée, pp. 87-88; Düttmann cite l'essai de Benjamin dans la traduction de Maurice de Gandillac, Mythe et violence, p. 81 .

${ }^{24}$ Sur les nombreuses affinités qui se dessinent entre la catharsis rigoureuse à laquelle Wittgenstein a soumis ses investigations en matière de langage et les mutations encourues par le langage poétique contemporain, notamment en ce qui a trait au regard qu'il porte sur les choses dans son usage de la langue, voir le bel ouvrage de Marjorie Perloff, Wittgenstein's Ladder. Poetic Language and the Strangeness of the Ordinary, Chicago, University of Chicago Press, 1996. 
cite le mot de Malebranche, d'après l'essai de Benjamin sur Kafka, selon lequel "l'attention est la prière naturelle de l'âme "; cette attention inconditionnelle qui, nous dit Celan, nourrit le « dialogue éperdu » auquel aspire le poème et qui ne tient qu'à " cette présence ponctuelle, unique - dans sa proximité immédiate même, elle concède à l'autre une parcelle de sa vérité : le temps de l'autre ${ }^{25}$. Cette attention soutenue qui, dans le poème, se noue dans un " retournement de souffle ", une Atemwende nouée entre inspir et expir, systole et diastole, marque aussi le passage au temps de l'autre dans la traduction, ce frayage intempestif de la parole étrangère dans la langue propre qui devient alors matrice d'une signifiance qu'elle portait à son insu, cela même qui ne se dit jamais, le " dire " lui-même qui en appelle à la gestation dans le nom de ce qui est sans nom. L'écart entre le "dire" et le "dit", ou encore l'asymétrie entre le "dire » et le "montrer" mise en évidence par Wittgenstein, est précisément ce qui s'ouvre et se referme dans l'acte de traduction, en tant qu'il lui est enjoint d'atteindre, de toucher, peut-être même seulement d'effleurer ce qui dans l' " essence langagière » se révèle comme pure communicabilité, où donc, l'espace d'un instant, l'unicité de la forme communique à sa propre expression l'étincelle qui donne lieu à ce contact fugitif, presque intangible, avec l'essence spirituelle des choses. C'est ainsi que Benjamin peut affirmer dans son désormais fameux mais non moins controversé essai sur la " tâche » ou, si on l'interprète et traduit à ma façon, l' « abandon » du traducteur :

Tout comme la tangente ne touche le cercle que de façon fugitive et en un seul point, et que c'est juste ce contact, non le point, qui lui assigne la loi selon laquelle elle poursuit à l'infini sa marche en ligne droite, de même la traduction touche-t-elle le sens de l'original de façon fugitive, et seulement en un point infiniment petit, pour de là suivre son propre cours selon la loi de fidélité dans la liberté du mouvement langagier. ${ }^{26}$

J'en viens donc à la seconde remarque. On conçoit bien que Benjamin en appelle à une notion de la traduction qui n'est absolument plus concernée par l'existence d'un signifié extérieur à la signifiance même

${ }^{25}$ Paul Celan, "Le méridien ", texte d'un discours prononcé à l'occasion de la remise du prix Georg Büchner en 1960, trad. par André du Bouchet, dans L'éphémère, $\mathrm{N}^{\mathrm{o}} 1,1965$, pp. 15-16.

${ }^{26}$ Trad. par L. Lamy et A. Nouss, dans TTR, Vol. X, N², 1997, p. 26. 
du nom, qui n'est pas alors un signifiant, un token ou un représentant de quelque chose, mais l'expression immédiate, sui generis, de ce partage unique où s'abrite et s'expose l'essence spirituelle du langage comme acte originel de dénomination. Ce partage qui, nous dit Benjamin, "se communique dans le langage et non par lui ", s'étend à tous les règnes, puisant dans la profondeur immémoriale d'un passé qui ne fut jamais présent, jusqu'à cet émoi très ancien qui aura départagé le pleur de l'animal du règne de la mort ${ }^{27}$, quand les paupières de l'homme se sont dessillées et que ses yeux sont devenus captifs de l'étendue balisée par le spectre de la lumière ontologique.

Que la communicabilité pure du nom soit incommunicable n'est pas l'effet d'une " opacité référentielle » (Quine) qui viendrait grever la transparence de l'énoncé, puisqu'on se situe en deçà de la propriété du dicible, des paramètres établis pour l'exercice d'un jugement sur l'être des choses. L'incommunicable est la communicabilité même, cet abîme qui s'ouvre et se referme quand la traduction touche à cette frontière intérieure de la langue dont la déchirure s'inscrit dans la trace de ce processus ininterrompu de métamorphoses qui la révèle à elle-même, dans l'inachèvement de sa forme, parfois dans une position d'extrême vulnérabilité, comme en témoignent certains poèmes de Paul Celan, évoluant à la limite de l'illisibilité. Cette rupture d'équilibre, qui est le moteur même de la vie, l'amorce d'une croissance inespérée là même où le pacte scellant la durée de tout ce qui tend à s'individuer semble consommé, ne tient pas tant à l'impossibilité de traduire le sens qui colle à la forme du signifiant original, mais au vertige naissant d'un possible qui s'ignorait dans la langue propre. Qu'est-ce à dire sinon que toute langue est originellement vouée à une extradition déportant la traduction qui s'y opère sur la ligne de front d'un continuum de métamorphoses qui répondent à l'exercice du langage comme forme de vie. Rien n'est définitif, même et surtout pour une existence rompue à l'aveu de finitude. Sur ce point, je ne saurais mieux m'exprimer que Benjamin dans ce passage de son essai Sur le langage en général et sur la langue des hommes, par lequel je vais clore mon exposé :

${ }^{27}$ À ce sujet, voir Giorgio Agamben, Le langage et la mort. Un séminaire sur le lieu de la négativité, trad. par Marilène Raiola, Paris, Christian Bourgois, 1991; on consultera notamment les pp. 83-94 sur l'analyse proposée par le jeune Hegel dans ses Leçons d'Iéna (Jenenser Realphilosophie), qui voit dans le cri de l'animal en danger de mort le point d'émergence de la subjectivité : " chaque animal a dans la mort violente une voix, il s'exprime soi-même en tant que soi supprimé [als aufgehobnes Selbst]. ” 
Die Übersetzung ist die Überführung der einen Sprache in die andere durch ein Kontinuum von Verwandlungen. Kontinua der Verwandlung, nicht abstrakte Gleichheits- und Ähnlichkeitsbezirke durchmisst die Übersetzung.

La traduction est le transfert d'une langue dans l'autre à travers un continuum de métamorphoses. La traduction traverse des continuums de métamorphose, non pas des zones abstraites d'équivalence et de ressemblance. $^{28}$

\section{Université de Montréal}

RÉSUMÉ : Le don et l'abandon : la trace de la traduction dans la blanche écriture du concept-Cette étude met en question l'ascendant du concept dans le domaine de l'expression ou, plus généralement, celui des " arts du sens ». L'impossibilité qui frappe la nécessité même de la traduction comme vecteur de la signification, loin de l'aliéner, l'instruit plutôt comme agent créateur d'une dimension de sens dont l'ouverture, virtuellement infinie, est intimement liée à la finitude qui s'attache au partage de la condition humaine. C'est là la limite du concept et le plan de démarcation de toute poétique en regard de la normativité inhérente à l'exercice du langage. C'est aussi la frontière qu'explore la traduction, qui ne saurait alors être reléguée à une fonction ancillaire en vertu de laquelle il lui incomberait simplement de préserver l'univocité du signifié (concept) commandant la translation idyllique entre une langue-source et une langue-cible. La traduction est par essence " performative ", c'est-à-dire qu'elle produit du sens. Ainsi fait-elle brèche vers une dimension d'universalité qui n'est pas d'abord subordonnée à l'unité du concept, mais se ressource et trouve son épanouissement dans l'unicité de la forme, celle d'une langue dont l'incomplétude est non seulement avérée, mais sollicitée dans son rapport à l'étranger. Il ne lui appartient pas de communiquer quelque chose, mais de révéler l'essence même de la communicabilité.

ABSTRACT : «Le don et l'abandon » : the Trace of Translation in the Stainless Writing of the Concept - This paper questions the ascendancy of the concept over the realm of expression and meaning. The impossibility which threatens the requirement of translation as

${ }^{28}$ W. Benjamin, "Über Sprache überhaupt und über die Sprache des Menschen ", dans Angelus Novus, Ausgewăhlte Schriften 2, Frankfurt am Main, Suhrkamp Taschenbuch, 1966, p. 20. 
vector of meaning assigns it rather as a creative agent of sense in a dimension whose open-ending, virtually infinite, is closely related to the finiteness of the human being. This is the limit of the concept and the starting-point of any poetics in its transgression of the normativity implied in language processing. This is also the frontier which is explored by translation, exceeding by the way the ancillary function of preserving the univocity of reference between a source-language and a target-language. Translation is essentially "performative" : it generates sense. The universal dimension of this performance is not regulated by the unity of the concept, but opened by the uniqueness of form, the one which emerges from the incompleteness of a singular language called for in its relation to the foreign. Translation has not to communicate something, but to reveal the very essence of communicability.

Laurent Lamy : Département de linguistique et de traduction, Université de Montréal, C.P. 6128, Succ. Centre-Ville, Montréal (Québec) H3C 3J7 